\title{
Estudo mineral das espécies vegetais Alternanthera brasiliana (L.) Kuntze e Bouchea fluminensis (Vell) Mold.
}

\author{
R.H. Delaporte ${ }^{1 *}$, K.P. Guzen ${ }^{1}$, O.S. Takemura ${ }^{1}$, J.C.P de Mello ${ }^{2}$ \\ ${ }^{1}$ Universidade Paranaense-UNIPAR, Praça Mascarenhas de Morais s/n, 87502-210, Umuarama, PR, Brasil, \\ ${ }^{2}$ Universidade Estadual de Maringá, Av. Colombo, 5790, 87020-900, Maringá, PR, Brasil

\begin{abstract}
RESUMO: Alternanthera brasiliana e Bouchea fluminensis são plantas encontradas amplamente na América do Sul, sendo utilizada pela população pelas suas propriedades analgésicas e antiinflamatórias. O trabalho objetivou determinar o teor de cinzas totais e os elementos minerais em ambas espécies. O teor de cinzas totais das folhas de $A$. brasiliana e $B$. fluminensis foi de 13,23\% $\pm 0,47$ e $8,28 \% \pm 0,07$, respectivamente. Em relação à presença de macro e micronutrientes nas folhas observou-se uma maior concentração de nitrogênio (3,13\%) e manganês $(0,296 \%)$ para $A$. brasiliana e de cálcio (3,08\%) e ferro (0,256\%) para B. fluminensis. Os dados obtidos, cinzas totais, macro e microelementos, contribuem significativamente no controle de qualidade e padronização de ambas drogas vegetais, além de dar suporte ao uso como suplemento nutricional.
\end{abstract}

Unitermos: Alternanthera brasiliana, Bouchea fluminensis, macronutrientes, micronutrientes, cinzas totais,

\begin{abstract}
Mineral evaluation of the species Alternanthera brasiliana (L.) Kuntze and Bouchea fluminensis (Vell) Mold.”. Alternanthera brasiliana and Bouchea fluminensis are plants widely distributed in South America. These plants are used by native populations as analgesic and anti-inflammatory agent. The aim of this paper was to assay the total ashes and mineral content in both species. The levels of total ashes in leaves of $A$. brasiliana and $B$. fluminensis were $13.23 \% \pm 0.47$ and $8.28 \% \pm 0.07$, respectively. The analysis of macro and micronutrients content in leaves of $A$. brasiliana shown as main components, nitrogen (3.13\%) and manganese (0.296\%). However, in B. fluminensis they were calcium (3.08\%) and iron $(0.256 \%)$. These data are very useful for the quality control and standardization of the plant raw materials, and support their use as nutritional agents.
\end{abstract}

Keywords: Alternanthera brasiliana, Bouchea fluminensis, macronutrients, micronutrients, total ashes.

\section{INTRODUÇÃO}

O uso de vegetais tem se difundido largamente nos últimos anos no tratamento de muitas doenças, na forma de medicamentos fitoterápicos. Por esta razão, existe um interesse crescente sobre o estudo da composição desses vegetais, tanto para constituintes orgânicos como para os inorgânicos (Lopes et al., 2002; Gomes et al., 2004). Além dos componentes orgânicos que apresentam outras propriedades medicinais, os macro e micronutrientes são importantes como suplementos nutricionais. Os macronutrientes podem favorecer a ativação de complexos enzimáticos fundamentais para processos bioquímicos vitais para o organismo humano. Entretanto, a alta concentração dos mesmos pode ser prejudicial. Por outro lado, os micronutrientes são importantes, pois podem ativar enzimas, como a angiotensina que, quando ativada pelo zinco, produz hipertensão, ou a peroxidação lipídica, quando ativada pelo ferro (Nappi; Vass, 2002).
Alternanthera brasiliana (L.) Kuntze (Amaranthaceae), conhecidapopularmenteno Brasil como "penicilina" e "perpétua do mato", tem sido utilizada na região sul do Brasil pelas suas propriedades analgésicas e antiinflamatórias (Reitz, 1972). Quanto aos estudos farmacológicos, a atividade analgésica foi avaliada por Macedo et al. (1999), a atividade antiedematogênica por Delaporte et al. (2001), e a atividade antiproliferativa de linfócitos por Brochado et al. (2003).

As folhas de $A$. brasiliana foram analisadas morfoanatomicamente por Delaporte et al. (2002b), Duarte; Debur (2004) e quimicamente por Macedo et al. (1999), Brochado et al. (2003) objetivando, respectivamente, o controle de qualidade e a identificação dos compostos químicos presentes nesta espécie.

A espécie Bouchea fluminensis (Vell.) Mold. é uma planta herbácea da família Verbenaceae difundida no Brasil e na Bolívia, sendo popularmente conhecida como "falso-gervão". Infusões das partes aéreas são utilizadas

ISSN 0102-695X 
na medicina popular como estimulante e reguladora do sistema digestivo (Corrêa, 1984). Estudos químicos descrevem o isolamento de alguns iridóides tais como: lamiídeo, durantosídeo e boucheosídeo (Schuquel et al., 1998) e de alguns triterpenóides como $\beta$-sitosterol e estigmasterol (Coube, 2000; Delaporte, 2001), além de um fenil-propanóide (Delaporte, 2001).

A atividade antiinflamatória e as análises farmacognósticas de Bouchea fluminesis, foram avaliadas por Coube (2000) e Delaporte et al. (2001). A substância majoritária identificada como lamiídeo, apresentou atividade antiinflamatória e antioxidante (Delaporte et al., 2002a). O extrato etanólico de $B$. fluminensis também mostrou um efeito analgésico central e periférico no modelo estudado por Costa et al. (2003).

Neste sentido, amostras de $A$. brasiliana e $B$. fluminensis foram analisadas com o propósito de se determinar os teores de cinzas totais e os elementos minerais (Mg, Ca, K, P, N, Fe, Cu, Mn, Zn, B e S), contribuindo no controle de qualidade e padronização das espécies, assim como, fornecer suporte para o seu uso na nutrição humana.

\section{MATERIAL E MÉTODOS}

As folhas de Alternanthera brasiliana e Bouchea fluminensis, foram coletadas no "Horto de plantas Medicinais" da Universidade Paranaense - UNIPAR (234’32” S; 54¹6’29” W) no verão/02 entre 8 e 10 h, sendo a colheita manual e utilizando as folhas adultas (limbo completamente expandido, exposto ao sol) cultivadas em canteiros experimentais, empregando adubação orgânica (humus de minhoca).

A espécie vegetal $A$. brasiliana foi identificada pela Dra. Elsie Guimarães (Herbário do Jardim Botânico do Rio de Janeiro) e um exemplar se encontra depositada no Herbário do Departamento de Biologia da Universidade Estadual de Maringá (4714 HUM). Já a espécie B. fluminensis foi identificada pela Dra. Fátima Regina Salimena-Pires e um exemplar foi depositado no Herbário da Universidade Federal de Juiz de Fora (CESJ 30113).

As folhas foram secas em estufa de ar circulante à $40{ }^{\circ} \mathrm{C}$. O teor de cinzas totais foi quantificado por meio de calcinação de $2 \mathrm{~g}$ de material seco em forno Mufla Quimis a $680-700{ }^{\circ} \mathrm{C}$ durante o período de $12 \mathrm{~h}$ e pesagem por $12 \mathrm{~h}$ após resfriamento em dessecador (Farmacopéia Brasileira, 1988). A metodologia adotada para a determinação de macro e micronutrientes, tanto nas folhas como nas cinzas totais, foi a técnica de mineralização descrita por Tedesco (1982), utilizando espectrofotômetro de absorção atômica GBC 932 plus.

\section{RESULTADOS E DISCUSSÃO}

Todas as análises foram realizadas em triplicata e o coeficiente de variação entre as medidas foi inferior a $5 \%$. Na Tabela 1 são apresentados os resultados obtidos de cinzas totais.

Tabela 1. Teor de cinzas totais das partes aéreas de ambas as espécies vegetais

\begin{tabular}{lccc}
\hline & \multicolumn{3}{c}{ Cinzas Totais } \\
\cline { 2 - 4 } Espécie Vegetal & $\mathrm{X}$ & $\delta$ & $\mathrm{CV}(\%)$ \\
\hline A. brasiliana & 13,23 & 0,47 & 3,56 \\
B. fluminensis & 8,28 & 0,07 & 0,89 \\
\hline
\end{tabular}

Legenda: $\mathrm{X}$ valor médio; $\delta$ desvio padrão; $\mathrm{CV}$ coeficiente de variação

Tabela 2. Teores médios de macronutrientes presentes nas folhas e nas cinzas totais de A. brasiliana e B. fluminensis

\begin{tabular}{ccccc}
\hline Macronutrientes & \multicolumn{2}{c}{ A. brasiliana } & \multicolumn{2}{c}{ B. fluminensis } \\
\cline { 2 - 4 }$(\%)$ & Folhas & Cinzas & Folhas & Cinzas \\
\hline $\mathrm{Mg}$ & 0,54 & 1,58 & 0,34 & 1,64 \\
$\mathrm{Ca}$ & 1,22 & 7,54 & 3,08 & 24,02 \\
$\mathrm{~K}$ & 2,35 & 5,62 & 1,02 & 4,68 \\
$\mathrm{~N}$ & 3,13 & 0,03 & 2,20 & 0,03 \\
$\mathrm{P}$ & 0,43 & 1,20 & 0,19 & 1,17 \\
\hline
\end{tabular}

Legenda: Mg: magnésio; Ca: cálcio; K: potássio; N: Nitrogênio; P: Potássio. 
Tabela 3. Teor médio de micronutrientes presentes nas folhas e nas cinzas totais de A.brasiliana e B. fluminensis

\begin{tabular}{ccccc}
\hline $\begin{array}{c}\text { Micronutrientes } \\
(\%)\end{array}$ & \multicolumn{2}{c}{ A. brasiliana } & \multicolumn{2}{c}{ B. fluminensis } \\
\cline { 2 - 5 } & Folhas & Cinzas & Folhas & Cinzas \\
\hline $\mathrm{Fe}$ & 0,139 & 0,338 & 0,256 & 1,927 \\
$\mathrm{Cu}$ & 0,011 & 0,128 & 0,007 & 0,131 \\
$\mathrm{Mn}$ & 0,296 & 0,513 & 0,022 & 1,451 \\
$\mathrm{Zn}$ & 0,091 & 1,750 & 0,127 & 3,720 \\
$\mathrm{~B}$ & 0,049 & 0,334 & 0,139 & 0,166 \\
$\mathrm{~S}$ & 0,16 & 0.64 & 0,14 & 0,87 \\
\hline
\end{tabular}

Legenda: Fe: ferro; Cu: cobre; Mn: manganês; Zn: zinco; B: boro; S: enxofre.

O conteúdo de cinzas totais estabelece a qualidade de substância residual não volátil no processo de incineração. As cinzas totais constituem-se das cinzas fisiológicas e não fisiológicas, quando se acrescenta ácido sulfúrico, as cinzas fisiológicas ou orgânicas são consumidas restando os constituintes silicosos e sílica presente no material (Círio et al., 2003). O conteúdo de cinzas encontrado para as plantas estudadas foi superior ao valor médio encontrado para outras drogas vegetais que se encontram na Farmacopéia Brasileira (Farmacopéia Brasileira, 1988).

A concentração dos macro e micronutrientes das partes aéreas de Alternanthera brasiliana e de Bouchea fluminensis encontram-se nas Tabela 2 e 3, respectivamente.

Em relação à presença de macro e micronutrientes nas folhas e cinzas das espécies, observa-se de uma forma geral, uma maior porcentagem destes nas cinzas, com exceção do nitrogênio. Isto provavelmente esteja relacionado com a existência de compostos nitrogenados orgânicos que fazem parte dos complexos enzimáticos, clorofilas e outras substâncias, que no processo de mineralização seriam perdidas como compostos voláteis e passam a não fazer parte das cinzas.

Como principal macronutriente das folhas de $A$. brasiliana se encontra o nitrogênio $(\mathrm{N})$ com concentração de $3,13 \%$, seguido do potássio (2,35\%) e do cálcio (1,22\%). Para as folhas de B. fluminensis o cálcio (3,08\%) foi o principal macronutriente, seguido pelo nitrogênio $(2,20 \%)$ e pelo potássio $(1,02 \%)$. Essa quantidade de cálcio, encontrada na amostra de $B$. fluminensis, quando utilizado em associação com outros alimentos, poderia ser considerado auxiliador nutricional para indivíduos com deficiência desse mineral (Lopes et al., 2001) (Tabela 2).

Nas cinzas, os principais macronutrientes encontrados foram o cálcio (7,54 e 24,02\%) e o potássio (5,62 e 4,68\%), para A. brasiliana e B. fluminensis, respectivamente.

Para as folhas de A. brasiliana (Tabela 3) o principal micronutriente foi o manganês $(0,30 \%)$ e em suas cinzas, o zinco (1,75\%). Por outro lado, nas folhas da $B$. fluminensis o principal micronutriente foi o ferro $(0,256 \%)$ seguido de boro $(0,139 \%)$ e o zinco $(0,127 \%)$, e em suas cinzas, o zinco e o ferro, com concentração de 3,72 e $1,92 \%$.

A presença de alguns elementos como cobre em grande quantidade poderia representar um risco tóxico aos usuários, assim como o alumínio e metais pesados (Almeida et al., 2002; Lopes et al., 2002). Entretanto no presente trabalho, a concentração deste elemento foi baixa nas duas espécies estudadas.

Estes resultados permitem confirmar que nas mais elevadas porcentagens de cinzas encontradas para ambas as espécies, os principais constituintes são os macronutrientes, fundamentalmente cálcio, potássio e magnésio, que não constituem elementos tóxicos ao organismo nas concentrações encontradas. Como conclusão pode-se sugerir que a presença destes nutrientes poderia indicar um possível uso destas plantas como suplemento nutricional.

\section{REFERÊNCIAS}

Almeida MMB, Lopes MFG, Nogueira CMD, Magalhães CEC, Morais NMT 2002. Determinação de nutrientes minerais em plantas medicinais. Ciênc Tecnol Aliment 22: 94-97.

Brochado CO, Almeida AP, Barreto BP, Costa LP, Ribeiro LS, Pereira RLC, Koatz VLG, Costa SS 2003. Flavonol robinobiosides and rutinosides from Alternanthera brasiliana (Amaranthaceae) and their effects on lymphocyte proliferation in vitro. $J$ Braz Chem Soc 14: 449-451.

Cirio GM, Doni Filho L, Miguel MD, Miguel OG, Zanin SMW 2003. Interrelação de parâmetros agronômicos e físicos de controle de qualidade de Maytenus iliciofolia, Mart ex. Reiss (Espinheira-santa) como insumo para indústria farmacêutica. Visão Acadêmica 4: 67-76.

Corrêa MP 1984. Dicionário de plantas úteis do Brasil e das exóticas cultivas. Rio de Janeiro: Imprensa Nacional.

Costa VB, Coube CS, Marinho BG, Matheus ME, Leitão 
SG, Fernandes SPD 2003. Anti-inflammatory and analgesic activity of Bouchea fluminensis. Fitoterapia 74: 364-371.

Coube CS 2000. Contribuição ao estudo farmacognóstico do falso gervão - Bouchea fluminensis Vell. Mold. Rio de Janeiro, 67p. Dissertação de Mestrado - Universidade Federal do Rio de Janeiro.

Delaporte RH, Sánchez GS, Cuellar AC, Mello JCP 2001. Control de calidad y actividad antiinflamatoria de las drogas vegetales Alternanthera brasiliana (L.) Kuntze y Bouchea fluminensis (Vell.) Mold. Acta Farm Bonaerense 20: 39-45.

Delaporte RH 2001. Estudio farmacognóstico, farmacológico y químico de Alternanthera brasiliana (L.) Kuntze y Bouchea fluminensis (Vell.) Mold. Havana - Cuba, 77p. Tese de doutorado - Universidade de Havana - Cuba.

Delaporte RH, Sánchez GS, Cuellar AC, Giuliani A, Mello JCP 2002a. Anti-inflammatory activity and lipid peroxidation inhition of iridoid lamiide isolated from Bouchea fluminensis (Vell.) Mold. (Verbenaceae). J Ethnopharmacol 82: 127-130.

Delaporte RH, Milaneze MA, Mello JCP, Jacomassi E. 2002b. Estudo farmacognóstico das folhas de Alternanthera brasiliana (L.) Kuntze (Amaranthaceae). Acta Farm Bonaerense 21: 169-174.

Duarte MR, Debur MC 2004. Characters of the leaf and steam morfo-anatomy of Alternanthera brasiliana (L.) Kuntze, Amaranthaceae. Revista Brasileira de Ciências Farmacêuticas 40: 85-92.

Farmacopéia Brasileira 1988. 4a. Ed. São Paulo: Atheneu.

Gomes MR, Soledad C, Olsina RA, Silva M, Martinez LD 2004. Metal content monitoring in Hypericum perforatum pharmaceutical derivatives by atomic absortion and emission spectrometry. J Pharm Biomed Anal 34: 569-576.

Lopes MFG, Almeida M, Nogueira CMD, Morais NMT 2001. Caracterização físico-química de algumas espécies de plantas medicinais. Rev Bras Pl Med 4: 95-98.

Lopes MFG, Almeida MB, Nogueira CMD, Morais NMT, Magalhães CEC 2002. Estudo mineral de plantas medicinais. Rev Bras Farmacogn 12: 115-116.

Macedo AF, Barbosa NC, Esquibel MA, Souza MM, CechinelFilho V 1999. Pharmacological and phytochemical studies of callus culture extracts from Alternanthera brasiliana. Pharmazie 54: 776-777.

Nappi JA, Vass E 2002. Interaction of iron with reactive intermediate of oxygen and nitrogen. Int $J$ Dev Neurosci 24: 134-142.

Reitz PR 1972. Amarantáceas: Flora Ilustrada Catarinense. São Paulo: Atheneu.

Schuquel ITA, Malheiros A, Sarragiotto MH 1998. Iridoid glusosides from Bouchea fluminensis. Phytochemistry 49: 2409-2411.

Tedesco MJ 1982. Extração simultânea de N, P, K, Ca e Mg em tecido de plantas por digestão de $\mathrm{H}_{2} \mathrm{O}_{2} \cdot \mathrm{H}_{2} \mathrm{SO}_{4}$. Porto Alegre: Departamento de solos/UFRGS. 